\title{
Introducing Anti-Vascular Endothelial Growth Factor Therapies for AMD Did Not Raise Risk of Myocardial Infarction, Stroke, and Death
}

Arseniy P. Yashkin, PhD, ${ }^{1}$ Paul Hahn, MD ${ }^{2}$ Frank A. Sloan, PhD ${ }^{1}$

Purpose: To assess the effect of availability of anti-vascular endothelial growth factor (VEGF) therapy on mortality and hospitalizations for acute myocardial infarction (AMI) and stroke over a 5-year follow-up period in United States Medicare beneficiaries newly diagnosed with exudative age-related macular degeneration (AMD) in 2006 compared with control groups consisting of beneficiaries (1) newly diagnosed with exudative AMD at a time when anti-VEGF therapy was not possible and (2) newly diagnosed with nonexudative AMD.

Design: Retrospective cohort study.

Participants: Beneficiaries newly diagnosed with exudative and nonexudative AMD in 2000 and 2006 selected from a random longitudinal sample of Medicare $5 \%$ claims and enrollment files.

Methods: Beneficiaries with a first diagnosis of exudative AMD in 2006 were the treatment group; beneficiaries newly diagnosed with exudative AMD in 2000 or nonexudative AMD in 2000 or 2006 were control groups. To deal with potential selection bias, we designed an intent-to-treat study, which controlled for nonadherence to prescribed regimens. The treatment group consisted of patients with clinically appropriate characteristics to receive anti-VEGF injections given that the therapy is available, bypassing the need to monitor whether treatment was actually received. Control groups consisted of patients with clinically appropriate characteristics but first diagnosed at a time when the therapy was unavailable (2000) and similar patients but for whom the therapy was not clinically indicated $(2000,2006)$. We used a Cox proportional hazard model.

Main Outcome Measures: All-cause mortality and hospitalization for AMI and stroke during follow-up.

Results: No statistically significant changes in probabilities of death and hospitalizations for AMI and stroke within a 5-year follow-up period were identified in exudative AMD beneficiaries newly diagnosed in 2006, the beginning of widespread anti-VEGF use, compared with 2000. As an alternative to our main analysis, which excluded beneficiaries from nonexudative AMD group who received anti-VEGF therapies during follow-up, we performed a sensitivity analysis with this group of individuals reincluded $(11 \%$ of beneficiaries newly diagnosed with nonexudative AMD in 2006). Results were similar.

Conclusions: Introduction of anti-VEGF agents in 2006 for treating exudative AMD has not posed a threat of increased risk of AMI, stroke, or all-cause mortality. Ophthalmology 2016;123:2225-2231 @ 2016 by the American Academy of Ophthalmology.

Supplemental material is available at www.aaojournal.org.

Anti-vascular endothelial growth factor (VEGF) therapies have been shown to be effective in slowing the progression of vision loss among persons diagnosed with exudative agerelated macular degeneration (AMD). ${ }^{1,2}$ Although the first anti-VEGF agent was approved in 2004, their use became widespread immediately after the introduction of bevacizumab (Genentech, San Francisco, CA) in 2006. Since then, anti-VEGF agents have become first-line therapy for the gamut of retinal vascular diseases. With the growth in use, there has been ongoing concern that anti-VEGF therapies and their extended administration may lead to increased risk of thromboembolic events. Some population-based studies have reported that anti-VEGF treatment of exudative AMD is associated with the development of coronary artery disease and stroke, ${ }^{3-7}$ or death associated with longterm use. ${ }^{8}$ Furthermore, it has been reported that $30 \%$ of persons with exudative AMD without a history of coronary artery disease have a high probability of acute atherothrombotic events. ${ }^{9}$ However, other studies have reported no association of anti-VEGF therapies with incident stroke, ${ }^{10-13}$ acute myocardial infarction (AMI), ${ }^{10,13-15}$ or short-term all-cause mortality. ${ }^{13,15-18}$

Although a recent summary of expert opinion concluded that these agents pose little systemic thromboembolic risk, ${ }^{19}$ 
the role of anti-VEGF therapy in mediating thromboembolic events is still insufficiently understood. ${ }^{20,21}$ Many prior assessments have lacked adequate power to detect statistically significant differences in these rare adverse outcomes, ${ }^{22}$ whereas others were based on the use of local, nonnationally representative samples and short follow-up periods after initiation of anti-VEGF therapy. An additional contributor to such inconclusive results may be the presence of selection bias in individuals treated with anti-VEGF agents. Although treatment with anti-VEGF agents is now common, a combination of issues including patient preferences leading to refusal of treatment, lack of local access, and presence of comorbidities and other conditions make use far from universal. Therefore, observed outcomes may reflect the process of selection into therapy rather than the effect of the therapy on health outcomes.

To deal with the potential problem of selection bias, we designed an intent-to-treat study. Intent-to-treat analysis is intended to control for the effect of nonadherence to prescribed regimens in randomized clinical studies. When applied to longitudinal health records data, in the context of our study, it allows for the treatment of all patients who have the clinically appropriate characteristics to receive antiVEGF injections, as the treatment group, bypassing the need to monitor whether treatment was actually received.

This study used a nationally representative $5 \%$ sample of all US Medicare beneficiaries enrolled in Medicare Parts A and $\mathrm{B}$ who were aged $68+$ years to assess the effect of the availability of anti-VEGF therapy on all-cause mortality and hospitalizations for stroke and AMI over a 5-year follow-up period in beneficiaries newly diagnosed with exudative AMD compared with beneficiaries newly diagnosed with exudative AMD at a time when anti-VEGF therapy was not possible.

\section{Methods}

\section{Data Sources}

Data came from a nationally representative 5\% random sample of claims filed between January 1, 1997, and December 31, 2013, on behalf of Medicare beneficiaries enrolled in Medicare Parts A and $\mathrm{B}$ and residing in the United States. Claims data were linked to an enrollment file providing information on enrollment type and status, dates of birth and death, and basic demographic information. The use of restricted Medicare claims and enrollment data was approved by Duke University's Institutional Review Board and adheres to the ethical principles of World Medical Association Declaration of Helsinki.

\section{Sample Selection}

The analysis sample consisted of Medicare beneficiaries first diagnosed with exudative or nonexudative AMD in 2000 and 2006 as recorded on at least 2 Medicare claims within 180 days of each other. Beneficiaries with a first diagnosis of exudative AMD in 2006 were the treatment group, and beneficiaries newly diagnosed with exudative AMD in 2000 or nonexudative AMD in 2000 or 2006 were used as control groups (Table 1). Analysis was restricted to beneficiaries aged 68+ years to allow for a 3-year look-back period, which was used to identify the date of the first exudative or nonexudative AMD diagnosis and to record
Table 1. List of Study Codes

\begin{tabular}{|c|c|c|}
\hline Condition & & Administrative Code* \\
\hline \multicolumn{3}{|l|}{ Dependent Variables } \\
\hline Myocardial infarction ${ }^{\dagger}$ & ICD-9: & 410.xx 412.xx \\
\hline Stroke $^{\dagger}$ & ICD-9: & 431.xx 434.xx 436.xx \\
\hline \multicolumn{3}{|l|}{ AMD } \\
\hline $\begin{array}{l}\text { Nonexudative senile } \\
\text { macular degeneration }\end{array}$ & ICD-9: & 362.51 \\
\hline $\begin{array}{l}\text { Exudative senile macular } \\
\text { degeneration }\end{array}$ & ICD-9: & 362.52 \\
\hline \multicolumn{3}{|l|}{ Exclusions } \\
\hline Retinal vein occlusion & ICD-9: & 362.35362 .36 \\
\hline Macular edema & ICD-9: & 362.07362 .53362 .83 \\
\hline \multicolumn{3}{|l|}{ Covariates } \\
\hline Diabetes mellitus, type 2 & ICD-9: & 250.xx \\
\hline Hypertension & ICD-9: & 401.xx \\
\hline Atrial fibrillation or flutter & ICD-9: & 427.31427 .32 \\
\hline Ischemic heart disease & ICD-9: & $\begin{array}{l}411.81411 .89414 .0 \times 414.8 \\
\quad 414.9\end{array}$ \\
\hline Angina & ICD-9: & 413.xx \\
\hline Congestive heart failure & ICD-9: & 402.x1 404.x1 428.xx 398.91 \\
\hline Cerebral ischemic attack & ICD-9: & 437.1 \\
\hline Transient ischemic attack & ICD-9: & 435.x \\
\hline $\begin{array}{l}\text { Intravitreal injection of } \\
\text { anti-VEGF agent }\end{array}$ & $\begin{array}{l}\text { CPT- } \\
\quad 4:\end{array}$ & $\begin{array}{l}67028 \text { followed by } 1 \text { of: J9035 } \\
\text { Q2024 C9257 C9399 J3490 } \\
\text { J3590 (bevacizumab } \\
\text { [Avastin; Genentech, Inc., } \\
\text { San Francisco, CA]), C9233 } \\
\text { J2778 (ranibizumab } \\
\text { [Lucentis; Genentech, Inc.]), } \\
\text { J2503 (pegaptanib [Macugen; } \\
\text { Valeant Pharmaceuticals, } \\
\text { Inc., Laval, Quebec, } \\
\text { Canada]), Q2046 C9291 } \\
\text { J0178 (aflibercept [Eylea; } \\
\text { Regeneron, Inc., Tarrytown, } \\
\text { NY]). }\end{array}$ \\
\hline
\end{tabular}

CPT $-4=$ Current Procedural Terminology 4th Edition; ICD-9 = International Classification of Diseases, 9th Revision; VEGF = vascular endothelial growth factor.

*Codes are drawn from ICD-9, Clinical Modification (ICD-9 for condition) and CPT-4 codes.

†Includes Part A inpatient claims only.

comorbidities. The date of the first claim with a confirmed study diagnosis was the date used for defining follow-up and look-back periods. The follow-up period was 5 years after the diagnosis date that qualified a beneficiary for inclusion in this study. The study outcomes, death and hospitalizations for stroke and AMI, were evaluated over the follow-up period. Beneficiaries diagnosed with macular edema or retinal vein occlusion over the 5-year follow-up period and beneficiaries with a hospitalization for stroke and/or myocardial infarction over the 3-year look-back period were excluded. In the primary analysis, beneficiaries in the 2006 nonexudative AMD control group who received antiVEGF therapies during the 5-year follow-up period were excluded $(11.1 \%$ ) (Table 2). A secondary sensitivity analysis with these individuals included also was performed. Most beneficiaries in the 2006 exudative AMD treatment group (53.3\%) received antiVEGF therapy at some time during the 5-year follow-up period after an initial diagnosis of exudative AMD. The 2000 and 2006 cohorts refer to the year of initial diagnosis, not to the year(s) of receipt of anti-VEGF injections, which could occur anytime during the 5-year follow-up period. After excluding beneficiaries 
Table 2. Sample Selection Process

\begin{tabular}{|c|c|c|c|c|}
\hline & \multicolumn{2}{|c|}{ Exudative AMD } & \multicolumn{2}{|c|}{ Nonexudative AMD } \\
\hline & 2006 & 2000 & 2006 & 2000 \\
\hline Initial sample pool & 8856 & 4540 & 12638 & 7727 \\
\hline \multicolumn{5}{|l|}{ Comorbidity restrictions } \\
\hline $\begin{array}{l}\text { Loss to retinal vein } \\
\text { occlusion }\end{array}$ & & & 782 & 541 \\
\hline Loss to macular edema & & & 2099 & 1297 \\
\hline $\begin{array}{l}\text { Sample pool after } \\
\text { comorbidity } \\
\text { restrictions }\end{array}$ & 8856 & 4540 & 9757 & 5889 \\
\hline $\begin{array}{l}\text { Receipt of anti-VEGF } \\
\text { within } 5 \text { yrs of } \\
\text { diagnosis }(\%)\end{array}$ & 53.26 & 0.00 & 11.14 & 0.00 \\
\hline \multicolumn{5}{|l|}{ Additional restrictions } \\
\hline Loss to VEGF injection & & & 1556 & 531 \\
\hline Loss to missing data & 2 & 1 & 2 & 0 \\
\hline Final sample size & 8854 & 4539 & 8199 & 5358 \\
\hline $\begin{array}{l}\text { Receipt of anti-VEGF } \\
\text { within } 5 \text { yrs of } \\
\text { diagnosis }(\%)\end{array}$ & 53.26 & 0.00 & 0.00 & 0.00 \\
\hline
\end{tabular}

$\mathrm{AMD}=$ age-related macular degeneration; $\mathrm{VEGF}=$ vascular endothelial growth factor.

diagnosed with macular edema and retinal vein occlusion, none of the beneficiaries in the 2000 groups (exudative or nonexudative AMD) received such therapy during the 5-year follow-up period.

\section{Statistical Analysis}

A Cox proportional hazard model, coupled with a difference-indifference explanatory variable design, was used to assess the relationships between availability of anti-VEGF therapies and the study outcomes. The difference-in-difference approach was based on the comparison of 4 mutually exclusive groups of beneficiaries. The treatment group consisted of beneficiaries newly diagnosed with exudative AMD in 2006. These individuals had clinical indications for anti-VEGF therapy and could receive it, if needed. The first control group consisted of beneficiaries newly diagnosed with exudative AMD in 2000. These persons had the same clinical indications for anti-VEGF therapy but could not receive the treatment because it was not available in 2000, and according to our calculations (Fig A1, Appendix, available at www.aaojournal.org) was used rarely even in the last year of the follow-up period for this cohort (2005). The technology became progressively more widely used in 2006 and in subsequent years. The last 2 control groups consisted of beneficiaries newly diagnosed with nonexudative AMD in 2000 and 2006, respectively. Anti-VEGF therapy is not clinically indicated for nonexudative AMD.

The general form of the equation to be estimated was $Y=\beta_{0}+\beta_{1} A+\beta_{2} T+\beta_{3} A * T+\beta_{4} X+\varepsilon$ (1), where $Y$ is the outcome of interest, $A$ is a binary indicator of anti-VEGF availability (i.e., for the 2006 cohort), $T$ is a binary indicator of the treatment group-exudative $\mathrm{AMD}, A * T$ is an interaction term, and $X$ is a matrix including all other covariates. The covariate $A$ measured temporal influences affecting the dependent variable applicable to both exudative and nonexudative AMD groups (e.g., changes in technology and practice patterns affecting beneficiaries newly diagnosed with exudative and nonexudative AMD in 2000 and 2006); $T$ measured time-invariant differences between the treatment and control groups (i.e., persistent differences between the treatment and control groups in study outcomes not attributable to availability of anti-VEGF injections). For example, one study found that the presence of AMD, especially exudative AMD, is prospectively associated with a higher risk of incident $\mathrm{AMI},{ }^{23}$ but another study found no difference in incident AMI. ${ }^{24}$ To our knowledge, no study has documented a difference between AMD and incident stroke. ${ }^{3,24}$ The interaction term $A * T$ measured the effect that the availability of anti-VEGF therapies to the treatment group had on the study outcomes. Specifically, $A * T$ represented the difference between 2 differences: [ $\left(Y_{T=2006, A=1}-\right.$ $\left.Y_{T=2000, A=1}\right)-\left(Y_{T=2006, A=0}-Y_{T=2000, A=0)]}\right.$ (2) the difference in study outcomes between beneficiaries newly diagnosed with exudative AMD in 2006/2000 minus the difference in such outcomes of beneficiaries newly diagnosed with nonexudative AMD in 2006/2000.

Covariates included in $X$ were male gender; black race; other race (white race, omitted reference group), age 75-84 years, age 85+ years (age 65-74 years omitted reference group); Charlson Index; and binary variables for prior diagnosis of diabetes mellitus, atrial fibrillation/flutter, ischemic heart disease, angina pectoris, congestive heart failure, cerebral ischemic attack, and transient ischemic attack (Table 1). Stata 11 (StataCorp 2009. Stata Statistical Software: Release 11. StataCorp LP, College Station, TX) and SAS 9.4 (SAS Institute Inc., Carey, NC) were used for the analysis.

\section{Results}

\section{Descriptive Sample Statistics}

Thirty-one percent of beneficiaries first diagnosed with exudative AMD in 2006 died within 5 years of diagnosis (Table 3). Death rates for beneficiaries diagnosed with nonexudative AMD were $4 \%$ lower than for contemporary beneficiaries diagnosed with exudative AMD. Death rates for the 5-year follow-up period were slightly higher (1\%) for the 2006 than the 2000 cohort. By contrast, rates of hospitalization for AMI and stroke during followup were slightly lower for the 2006 than the 2000 cohorts. The majority of sample persons were white women in both years with substantial increases in the proportions of women older than 85 years of age in 2006. The mean value of the Charlson Index, a measure of general health, was higher for beneficiaries with exudative AMD than for those with nonexudative AMD, indicating that exudative AMD was associated with substantially worse general health. Although the general health of persons with exudative AMD tended to decrease between 2000 and 2006, the opposite pattern occurred for beneficiaries with nonexudative AMD. This decline in general health of newly diagnosed exudative AMD beneficiaries was not generally reflected in the specific comorbidities analyzed in this study (prior diagnoses of cardiovascular and cerebrovascular conditions other than AMI or stroke and diabetes mellitus), which demonstrated a modest decrease in prevalence in 2006.

\section{Cox Proportional Hazard Analysis}

No statistically significant changes in the probabilities of death and hospitalizations for AMI and stroke within a 5-year followup period were identified in exudative AMD beneficiaries newly diagnosed in 2006, the beginning of widespread anti-VEGF use, compared with 2000 (Table 4). The key results are for the estimates for the difference-in-difference hazard ratio (HR) corresponding to $\beta_{3}$ from Equation 1 as described earlier. On the basis of these HRs, probabilities of adverse study health outcomes among exudative AMD beneficiaries in 2006 compared with 2000 declined by $8 \%$ for all-cause mortality (HR, 0.92; $95 \%$ confidence interval [CI], 0.84-1.01), $10 \%$ for AMI (HR, 0.90; 
Table 3. Summary Statistics

\begin{tabular}{|c|c|c|c|c|}
\hline & Exudative 2006 & Exudative 2000 & Nonexudative 2006 & Nonexudative 2000 \\
\hline Death & $0.31(0.46)$ & $0.30(0.46)$ & $0.27 * * *(0.45)$ & $0.26 * * *(0.44)$ \\
\hline Myocardial infarction & $0.04(0.20)$ & $0.06 * * *(0.24)$ & $0.04(0.20)$ & $0.05 * *(0.23)$ \\
\hline Stroke & $0.05(0.22)$ & $0.07 * * *(0.25)$ & $0.05(0.22)$ & $0.06 *(0.23)$ \\
\hline Male & $0.22(0.41)$ & $0.34 * * *(0.47)$ & $0.19 * * *(0.40)$ & $0.33 * * *(0.47)$ \\
\hline Black & $0.01(0.11)$ & $0.01(0.10)$ & $0.02 * * *(0.14)$ & $0.02 * * *(0.14)$ \\
\hline Other race & $0.02(0.14)$ & $0.02(0.14)$ & $0.03 *(0.16)$ & $0.02(0.15)$ \\
\hline Age: $75-84$ yrs & $0.50(0.50)$ & $0.51(0.50)$ & $0.49(0.50)$ & $0.53 * * *(0.50)$ \\
\hline Age: $85+$ yrs & $0.36(0.48)$ & $0.27 * * *(0.45)$ & $0.33 * * *(0.47)$ & $0.25 * * *(0.43)$ \\
\hline Charlson Index & $1.38(1.86)$ & $1.17^{* * *}(1.74)$ & $0.15 * * *(0.75)$ & $0.32 * * *(1.00)$ \\
\hline Diabetes mellitus & $0.13(0.34)$ & $0.14(0.35)$ & $0.14 *(0.35)$ & $0.15 * * *(0.36)$ \\
\hline Hypertension & $0.26(0.44)$ & $0.40 * * *(0.49)$ & $0.33 * * *(0.47)$ & $0.40 * * *(0.49)$ \\
\hline Atrial fibrillation/flutter & $0.09(0.29)$ & $0.12 * * *(0.33)$ & $0.10(0.30)$ & $0.12 * * *(0.33)$ \\
\hline Ischemic heart disease & $0.12(0.33)$ & $0.25 * * *(0.43)$ & $0.15 * * *(0.36)$ & $0.24 * * *(0.43)$ \\
\hline Angina & $0.04(0.21)$ & $0.13 * * *(0.33)$ & $0.05(0.21)$ & $0.11 * * *(0.31)$ \\
\hline Congestive heart failure & $0.11(0.31)$ & $0.18 * * *(0.39)$ & $0.10(0.30)$ & $0.17 * * *(0.37)$ \\
\hline Cerebral ischemic attack & $0.02(0.15)$ & $0.01 * *(0.12)$ & $0.02(0.15)$ & $0.02 *(0.13)$ \\
\hline Transient ischemic attack & $0.05(0.21)$ & $0.09 * * *(0.29)$ & $0.04(0.20)$ & $0.09 * * *(0.28)$ \\
\hline $\mathrm{N}$ & 8854 & 4539 & 8199 & 5358 \\
\hline
\end{tabular}

Data shown are the sample means with standard deviations in parentheses.

Asterisks show the results for $t$ tests with exudative AMD 2006 as the comparison group: *Significant at $\alpha \leq 0.05$. **Significant at $\alpha \leq 0.01 . * * *$ Significant at $\alpha \leq 0.001$.

$95 \% \mathrm{CI}, 0.72-1.12$ ), and $17 \%$ for stroke (HR, $0.83 ; 95 \% \mathrm{CI}$, 0.67-1.02). However, none of these decreases were statistically significant. Likewise, by comparing exudative with nonexudative AMD beneficiaries, trends of increased death, AMI, and stroke were identified in exudative AMD beneficiaries, but none of the HRs were statistically significant. A statistically significant increase in mortality rates was identified in both newly diagnosed exudative and nonexudative beneficiaries as a combined group in 2006 compared with 2000 (HR, 1.09; 95\% CI, 1.02-1.17). The implied increase in the probability of death for beneficiaries newly diagnosed with AMD was higher than indicated by the changes in the mean values reported in Table 3. Table 4 reports the results of multivariate analysis; there were substantial changes in case-mix between the 2000 and 2006

Table 4. Survival Functions for Mortality, Myocardial Infarction, and Stroke

\begin{tabular}{|c|c|c|c|}
\hline & Mortality & Myocardial Infarction & Stroke \\
\hline Exudative AMD×2006 & $0.92[0.84-1.01]$ & $0.90[0.72-1.12]$ & $0.83[0.67-1.02]$ \\
\hline Exudative $\mathrm{AMD}^{8}$ & $1.03[0.95-1.11]$ & $1.04[0.88-1.23]$ & $1.09[0.93-1.28]$ \\
\hline $2006^{\|}$ & $1.09 *[1.02-1.17]$ & $0.87[0.74-1.02]$ & $0.88[0.76-1.03]$ \\
\hline Male & $1.25 * * *[1.19-1.32]$ & $1.24 * * *[1.09-1.40]$ & 0.97 [0.86-1.09] \\
\hline Black & $1.14[0.96-1.36]$ & $1.23[0.83-1.83]$ & $1.12[0.75-1.68]$ \\
\hline Other race & $0.84 *[0.70-0.99]$ & $0.58 *[0.36-0.93]$ & $1.05[0.74-1.48]$ \\
\hline Age: $75-84$ yrs & $1.98 * * *[1.81-2.16]$ & 1.39 *** $[1.17-1.65]$ & $1.58 * * *[1.34-1.87]$ \\
\hline Age: $85+$ yrs & $4.59 * * *[4.21-5.00]$ & $2.11 * * *[1.77-2.52]$ & $2.48 * * *[2.09-2.96]$ \\
\hline Charlson Index & $1.12 * * *[1.11-1.14]$ & $1.07 * * *[1.04-1.11]$ & $1.07 * * *[1.03-1.11]$ \\
\hline Diabetes mellitus & $1.11 * *[1.04-1.18]$ & $1.19 *[1.03-1.38]$ & $1.09[0.94-1.26]$ \\
\hline Hypertension & $0.95 *[0.90-0.99]$ & $0.96[0.85-1.08]$ & $1.00[0.90-1.12]$ \\
\hline Atrial fibrillation/flutter & 1.36 *** [1.28-1.45] & $1.15[0.97-1.35]$ & $1.40 * * *[1.20-1.63]$ \\
\hline Ischemic heart disease & $1.09 * *[1.02-1.15]$ & $1.17 *[1.01-1.34]$ & $1.12[0.98-1.28]$ \\
\hline Angina & $0.99[0.91-1.08]$ & $1.38 * * *[1.15-1.65]$ & $1.02[0.85-1.24]$ \\
\hline Congestive heart failure & $1.52 * * *[1.43-1.61]$ & $1.64 * * *[1.42-1.89]$ & $1.06[0.92-1.24]$ \\
\hline Cerebral ischemic attack & $1.23 * *[1.07-1.41]$ & $1.24[0.88-1.74]$ & $1.68 * * *[1.28-2.20]$ \\
\hline Transient ischemic attack & $1.17 * * *[1.08-1.28]$ & $1.08[0.87-1.33]$ & $1.75 * * *[1.48-2.07]$ \\
\hline $\mathrm{N}$ & 26950 & $26608^{\dagger}$ & $26679^{\dagger}$ \\
\hline
\end{tabular}

$\mathrm{AMD}=$ age-related macular degeneration.

*Significant at $\alpha \leq 0.05$. $* *$ Significant at $\alpha \leq 0.01$. $* * *$ Significant at $\alpha \leq 0.001$.

† Sample size reduced because of instances of outcome of interest before baseline diagnosis.

${ }^{\ddagger}$ Exudative AMD (2006) $=1$ versus all other 3 AMD categories $=0$.

${ }^{\S}$ Exudative AMD $(2006+2000)=1$ versus nonexudative AMD $(2006+2000)=0$.

"Exudative AMD (2006) + nonexudative AMD (2000) = 1 versus exudative AMD (2006) + nonexudative AMD (2006) = 1 versus exudative AMD $(2000)+$ nonexudative AMD $(2000)=0$. 
Yashkin et al • AMI, Stroke, Mortality Post Anti-VEGF

Table 5. Sensitivity Testing of Key Covariates

\begin{tabular}{|c|c|c|c|c|c|c|}
\hline & \multicolumn{3}{|c|}{ Main Specification } & \multicolumn{3}{|c|}{ Alternate Specification ${ }^{\dagger}$} \\
\hline & Mortality & Myocardial Infarction & Stroke & Mortality & Myocardial Infarction & Stroke \\
\hline Exudative AMD $\times 2006^{\ddagger}$ & $0.92[0.84-1.01]$ & $0.90[0.72-1.12]$ & $0.83[0.67-1.02]$ & $0.97[0.88-1.06]$ & $0.91[0.74-1.13]$ & $0.86[0.70-1.05]$ \\
\hline Exudative AMD & $1.03[0.95-1.11]$ & $1.04\left[\begin{array}{ll}0.88 & 1.23\end{array}\right]$ & $1.09[0.93-1.28]$ & $1.15^{* * * *}\left[\begin{array}{ll}1.06 & 1.24\end{array}\right]$ & $1.07[0.91-1.26]$ & $1.16[0.99-1.36]$ \\
\hline 2006 & $1.09 *[1.02-1.17]$ & 0.87 [0.74-1.02] & $0.88[0.76-1.03]$ & $1.04[0.97-1.11]$ & $0.86^{*}[0.74-1.00]$ & $0.87 *[0.75-1.00]$ \\
\hline $\mathrm{N}$ & 26950 & 26608 & 26679 & 29037 & 28662 & 28749 \\
\hline
\end{tabular}

$*$ Significant at $\alpha \leq 0.05$. ***Significant at $\alpha \leq 0.001$.

${ }^{\dagger}$ In the alternate specification, the nonexudative AMD groups include people who received anti-VEGF treatment. The main specification repeats from Table 4.

${ }^{\ddagger}$ Exudative AMD $(2006)=1$ versus all other 3 AMD categories $=0$.

${ }^{\S}$ Exudative AMD $(2006+2000)=1$ versus nonexudative AMD $(2006+2000)=0$.

$\|$ Exudative AMD (2006) + nonexudative AMD $(2000)=1$ versus exudative AMD (2006) + nonexudative AMD (2006) $=1$ versus exudative AMD $(2000)+$ nonexudative AMD $(2000)=0$.

cohorts, in particular, the decrease in the mean value of the Charlson index for the nonexudative AMD group.

Results for the other covariates were plausible, with the exception of hypertension in the analysis of all-cause mortality. A diagnosis of hypertension at baseline was associated with reduced mortality during follow-up (HR, 0.95; 95\% CI, 0.90-0.99), which may in part reflect active management of hypertension in beneficiaries with a diagnosis of hypertension compared with those beneficiaries without diagnosed hypertension who presumably were not treated for this condition.

As an alternative to our main analysis, which excluded beneficiaries who received anti-VEGF therapies from the nonexudative AMD group, we performed a sensitivity analysis with this group of individuals reincluded (Table 5). In the alternate specification, the HRs comparing study outcomes of beneficiaries newly diagnosed with exudative AMD in 2006 versus $2000\left(\beta_{3}\right)$ generally increased slightly but were still not statistically significant. The HR for the risk of all-cause mortality among exudative AMD versus nonexudative AMD beneficiaries (2006 and 2000, inclusive) became larger (HR, 1.15; 95\% CI, 1.06-1.24) and statistically significant. In addition, the HR for risk of AMI and stroke in 2006 versus 2000 also became significant, but these results, as in Table 3, imply that, after accounting for other factors, the 2006 cohorts faced a lower risk of AMI and stroke than the 2000 cohorts did. The HR for the binary variable for the 2006 cohort fell in the alternative specification and in contrast to the main specification was no longer statistically significant.

\section{Discussion}

Our analysis of individuals newly diagnosed with exudative AMD showed that widespread use of anti-VEGF agents in 2006 for the treatment of this condition did not lead to increased mortality or hospitalizations for AMI and stroke in this population of individuals.

Attributes of beneficiaries newly diagnosed with both AMD types were often similar with the notable exception of the Charlson Index, a marker for general health, which was worse among exudative AMD beneficiaries. There were no statistically significant differences in incident AMI or stroke between beneficiaries newly diagnosed with exudative compared with nonexudative AMD.

We accounted for differences in beneficiary attributes by including them as covariates in our multivariate analysis.
Likewise, the analysis accounted for intertemporal changes common to both AMD types by combining data on beneficiaries newly diagnosed with these conditions in 2000 before anti-VEGF agents were available with data from 2006, the first year when such agents were widely used. This study extended another intent-to-treat analysis based on the same data that assessed changes in vision outcomes ${ }^{25}$ after widespread use of anti-VEGF agents for treating exudative AMD. $^{26}$

\section{Study Limitations and Strengths}

We used an intent-to-treat design to overcome selection bias inherent in clinical treatment of exudative AMD. It was hypothesized and then observed that individuals receiving anti-VEGF therapy tended to be healthier than their counterparts who did not undergo the treatment, indicating the presence of significant selection bias toward avoiding treatment in those whose systemic comorbidities may be thought to override any benefit of anti-VEGF treatment. Our intent-to-treat study design, which allowed for the assessment of health outcomes for the entire sample of Medicare beneficiaries newly diagnosed with exudative AMD rather than just those receiving anti-VEGF treatment, was used to overcome this limitation.

This study has several other important strengths, including the national representativeness of the data, the analysis sample sizes, which are larger than in many previous studies, the long follow-up period, and the use of the intent-to-treat design to deal with selection in use of the study therapy.

We also acknowledge some study limitations. First, even after screening, slightly more than $10 \%$ of beneficiaries in the 2006 control group of newly diagnosed nonexudative AMD beneficiaries received anti-VEGF therapy during the 5-year follow-up period. These beneficiaries were diagnosed with exudative AMD at the time they received anti-VEGF therapy during follow-up. They were not diagnosed with exudative AMD in 2006, and therefore were not part of the treatment group. Nevertheless, we cannot rule out coding errors at baseline, that is, that beneficiaries had exudative AMD in 2006, but the claims did not indicate this. The issue of coding errors reflects a broader issue of errors in coding 
of diagnoses in claims data, which were not designed for use in research. Second, we did not evaluate the relationship between specific anti-VEGF agents and AMI, stroke, and mortality outcomes. Third, although concerns about adverse effects of anti-VEGF therapies in the existing literature and in this study have largely focused on potential adverse outcomes to the cardiovascular and cerebrovascular systems, there may be other potential adverse outcomes, such as Parkinson's disease, which were not evaluated. ${ }^{27}$ Finally, we studied the impact of the introduction of anti-VEGF therapies on elderly persons newly diagnosed with exudative AMD, not just those who received such therapies, to minimize effects from treatment selection bias. However, in an additional sensitivity analysis not presented, we compared persons who actually received the therapies with others who did not and found that the former group surprisingly fared better than the latter, which we attributed to selection. Although our analysis indicated no adverse effects on all-cause mortality, AMI, or stroke on average, we cannot rule out the possibility that some individuals who received relatively large numbers of injections were more prone to experience adverse outcomes of the types we analyzed.

In summary, with the caveats just noted, data from this study do not show that the introduction of anti-VEGF agents in 2006 for treating exudative AMD has posed a threat of increased risk of AMI, stroke, or all-cause mortality.

\section{References}

1. Skaat A, Chetrit A, Belkin M, et al. Time trends in the incidence and causes of blindness in Israel. Am J Ophthalmol 2012;153:214-21.

2. Bloch SB, Larsen M, Munch IC. Incidence of legal blindness from age-related macular degeneration in Denmark: year 2000 to 2010. Am J Ophthalmol 2012;153:209-13.

3. Sun C, Klein R, Wong TY. Age-related macular degeneration and risk of coronary heart disease and stroke: the Cardiovascular Health Study. Ophthalmology 2009;116:1913-9.

4. Hu C-C, Ho J-D, Lin H-C. Neovascular age-related macular degeneration and the risk of stroke a 5-year population-based follow-up study. Stroke 2010;41:613-7.

5. Ikram MK, Mitchell P, Klein R, et al. Age-related macular degeneration and long-term risk of stroke subtypes. Stroke 2012;43:1681-3.

6. Pratt NL, Ramsay EN, Kemp A, et al. Ranibizumab and risk of hospitalisation for ischaemic stroke and myocardial infarction in patients with age-related macular degeneration: a self-controlled case-series analysis. Drug Safety 2014;37: 1021-7.

7. Schlenker MB, Thiruchelvam D, Redelmeier DA. Intravitreal anti-vascular endothelial growth factor treatment and the risk of thromboembolism. Am J Ophthalmol 2015;160: 569-80.

8. Avery RL, Gordon GM. Systemic safety of prolonged monthly anti-vascular endothelial growth factor therapy for diabetic macular edema a systematic review and meta-analysis. JAMA Ophthalmol 2016;134:21-9.

9. Taniguchi H, Shiba T, Maeno T, Takahashi M. Evaluation of carotid atherosclerosis, peripheral arterial disease, and chronic kidney disease in patients with exudative age-related macular degeneration without coronary artery disease or stroke. Ophthalmologica 2015;233:128-33.

10. Curtis LH, Hammill BG, Schulman KA, Cousins SW. Risks of mortality, myocardial infarction, bleeding, and stroke associated with therapies for age-related macular degeneration. Arch Ophthalmol 2010;128:1273-9.

11. Tunon J, Maria Ruiz-Moreno J, Luis Martin-Ventura J, et al. Cardiovascular risk and antiangiogenic therapy for agerelated macular degeneration. Surv Ophthalmol 2009;54: 339-48.

12. Fernandez AB, Panza GA, Cramer B, et al. Age-related macular degeneration and incident stroke: a systematic review and meta-analysis. PLoS One 2015;10:e0142968.

13. Ng WY, Tan GSW, Ong P-G, et al. Incidence of myocardial infarction, stroke, and death in patients with age-related macular degeneration treated with intravitreal anti-vascular endothelial growth factor therapy. Am J Ophthalmol 2015;159: 557-64.

14. Campbell RJ, Bell CM, Paterson JM, et al. Stroke rates after introduction of vascular endothelial growth factor inhibitors for macular degeneration: a time series analysis. Ophthalmology 2012;119:1604-8.

15. Kitchens JW, Do DV, Boyer DS, et al. Comprehensive review of ocular and systemic safety events with intravitreal aflibercept injection in randomized controlled trials. Ophthalmology 2016;123:1511-20.

16. French DD, Margo CE. Age-related macular degeneration, anti-vascular endothelial growth factor agents, and short-term mortality: a postmarketing medication safety and surveillance study. Retina 2011;31:1036-42.

17. Ueta T, Noda Y, Toyama T, et al. Systemic vascular safety of ranibizumab for age-related macular degeneration. Ophthalmology 2014;121:2193-203.

18. Schmidt-Erfurth U. Clinical safety of ranibizumab in agerelated macular degeneration. Expert Opin Drug Saf 2010;9: 149-65.

19. Semeraro F, Morescalchi F, Duse S, et al. Systemic thromboembolic adverse events in patients treated with intravitreal anti-VEGF drugs for neovascular age-related macular degeneration: an overview. Expert Opin Drug Saf 2014;13: $785-802$.

20. Dedania VS, Bakri SJ. Systemic safety of intravitreal antivascular endothelial growth factor agents in age-related macular degeneration. Curr Opin Ophthalmol 2016;27: 224-43.

21. Csaky K, Do DV. Safety implications of vascular endothelial growth factor blockade for subjects receiving intravitreal antivascular endothelial growth factor therapies. Am J Ophthalmol 2009;148:647-56.

22. Thulliez M, Angoulvant D, Le Lez ML, et al. Cardiovascular events and bleeding risk associated with intravitreal antivascular endothelial growth factor monoclonal antibodies: systematic review and meta-analysis. JAMA Ophthalmol 2014;132:1317-26.

23. Duan Y, Mo J, Klein R, et al. Age-related macular degeneration is associated with incident myocardial infarction among elderly Americans. Ophthalmology 2007;114:732-7.

24. Alexander SL, Linde-Zwirble WT, Werther W, et al. Annual rates of arterial thromboembolic events in Medicare neovascular age-related macular degeneration patients. Ophthalmology 2007; 114:2174-8.

25. Sloan FA, Hanrahan BW. The effects of technological advances on outcomes for elderly persons with exudative agerelated macular degeneration. JAMA Ophthalmol 2014;132: 456-63. 
26. Stein JD, Hanrahan BW, Comer GM, Sloan FA. Diffusion of technologies for the care of older adults with exudative age-related macular degeneration. Am J Ophthalmol 2013;155:688-96.
27. Chung S-D, Ho J-D, Hu C-C, et al. Increased risk of Parkinson disease following a diagnosis of neovascular age-related macular degeneration: a retrospective cohort study. Am J Ophthalmol 2014;157:464-9.

\section{Footnotes and Financial Disclosures}

Originally received: April 26, 2016.

Final revision: June 20, 2016.

Accepted: June 21, 2016.

Available online: August 11, 2016.

Manuscript no. 2016-871.

${ }^{1}$ Department of Economics, Duke University, Durham, North Carolina.

${ }^{2}$ NJRetina, Morristown, New Jersey.

Financial Disclosure(s):

The author(s) have made the following disclosure(s): P.H.: Consulting agreement unrelated to the submitted work with Second Sight Medical Products and Bausch \& Lomb.

Supported in part by the National Institute on Aging (grant R01AG017473). The sponsor or funding organization had no role in the design or conduct of this research.

Author Contributions:

Conception and design: Hahn, Sloan
Data collection: Sloan

Analysis and interpretation Yashkin, Hahn, Sloan

Obtained funding: Sloan

Overall responsibility: Yashkin, Hahn, Sloan

Abbreviations and Acronyms:

AMD = age-related macular degeneration; AMI $=$ acute myocardial infarction; $\mathbf{C I}=$ confidence interval; $\mathbf{H R}=$ hazard ratio; $\mathbf{V E G F}=$ vascular endothelial growth factor.

Correspondence:

Frank A. Sloan, PhD, Department of Economics, Duke University, 213 Social Sciences Building, Box 90097, Durham, NC 27708. E-mail: fsloan@ duke.edu. 